\title{
Viscous oscillations in a circular cylinder with an elastic cover on the free surface
}

\author{
Rangachari Kidambi
}

Received: 4 April 2008 / Accepted: 31 January 2009 / Published online: 18 February 2009

\begin{abstract}
The effects of an elastic membrane on the viscous oscillations of liquid filling a circular cylindrical container are studied by using the natural viscous complex eigenfunctions of the problem. The free surface of the liquid is assumed to be fully covered by the membrane. By projecting the governing equations onto an appropriate basis, a nonlinear eigenvalue problem for the complex frequencies is obtained. This is then solved to obtain the modal frequencies as a function of the Reynolds number Re, the tension parameter $\tau$, the mass parameter $\zeta$ and the liquid depth $h$. The zero velocity conditions on both the side and bottom walls are satisfied unlike in earlier studies where either only the sidewall or only the bottom wall conditions were met. Results are presented for the four lowest non-axisymmetric modes as a function of $\operatorname{Re}, h, \tau$ and $\zeta$. The elastic cover increases the slosh frequencies but only in comparison with an uncovered free surface with a contact line that is free to move; the frequencies are lower when compared with those of a free surface with pinned contact line. There are ranges of $\operatorname{Re}, h, \tau$ and $\zeta$ for which the oscillations are overdamped and the sloshing is aperiodic. Though the frequencies and damping rates decrease for an increasing mass of the elastic cover, there exist ranges of $\mathrm{Re}, h$ and $\tau$ for which the heavier cover produces higher slosh frequencies.
\end{abstract}

Keywords Circular cylinder - Complex eigenfunctions · Hydroelastic oscillations - Non-axisymmetric modes · Nonlinear eigenvalue problem

\section{Introduction}

The use of large and light-weight tank structures in aerospace vehicles has led to an extensive study of their stability and control. This is a difficult problem as the interaction of the fluid and tank structure has to be taken into account. Some of these studies have focused on the fluid interaction with the elastic cylinder wall (see references in [1]) while others have investigated the effects of an elastic bottom (see references in [1]). Meanwhile various devices like liquid or movable surface devices (floating lids/mats, floating cans) and fixed baffles have been employed to suppress slosh and move the coupled frequencies away from the control [2]. It is important to know the frequencies and damping rates of these coupled systems. In this paper, we determine the frequencies and damping rates of one

R. Kidambi (凶)

Computational \& Theoretical Fluid Dynamics Division, National Aerospace Laboratories, Bangalore 560017, India

e-mail: kidambi@ctfd.cmmacs.ernet.in 
such system consisting of a liquid in a circular cylindrical container with the free surface of the liquid covered by an elastic cover.

Though apparently a linear problem that has been studied for the last fifty years, a complete solution has not been published, to our knowledge. For instance, the recent paper [1] presents a method that works only for large liquid depths, ignoring as it does the no-slip condition on the bottom wall. Bauer and Chiba [3] present another method for the same problem; however, the applicability in this case is only to small liquid depths. Analogous studies for axisymmetric oscillations have been published in [4]. In all these studies, real eigenfunctions are used to represent the velocity and pressure fields and these representations do not turn out to be rich enough to satisfy all boundary conditions. Indeed, Bauer and Chiba [1] write 'The analytical approaches of shallow and large-aspect-ratio containers cannot, due to the different analytical procedures, yield an acceptable transition region $h / a$ '.

The present approach overcomes these shortcomings by employing the natural complex eigenfunctions of the linearised Navier-Stokes equations for the cylindrical geometry to represent the velocities and pressures; these representations turn out to be rich enough to satisfy all boundary conditions simultaneously. This method was devised to obtain a semi-analytic solution to the unsteady Stokes problem in a lid-driven cylindrical container [5]; it has recently been applied to the damping of viscous oscillations in a cylindrical container with pinned contact line [6]. In this paper, the method is appropriately modified to take into account the elastic surface cover. For numerous examples of the usage of complex eigenfunction expansions to calculate slow viscous flows, see [7].

The coupled frequencies and damping rates for any liquid depth, with all boundary conditions satisfied, can be obtained by the present method. The results from the current method for the case of no elastic cover are in excellent agreement with extensive experimental results of Howell et al. [8]. However, there is a large discrepancy between the present results and those in [1] and [3].

The paper is organised as follows. Section 2 presents the governing equations and boundary conditions. Section 3 presents an eigenvalue formulation and solution of the problem where the natural viscous complex eigenfunctions of the problem are used. Section 4 presents results for a range of Reynolds number, liquid depth and mass and tension parameters; these include comparisons with published analytical [9], experimental [8] and numerical results of [1] and [3]. The variations of the frequency and damping rate with the mass parameter $\zeta$, the tension parameter $\tau$, liquid depth $h$ and the Reynolds number Re are also explored for the lowest slosh modes.

\section{Governing equations}

We consider the small oscillations of viscous liquid filling a circular cylindrical container of radius $R$; an elastic membrane is assumed to fully cover the free surface. In this paper, we use 'elastic cover' and 'elastic membrane' interchangeably. Figure 1 shows the geometry and coordinate system. Scaling lengths by $R$ and time by $\sqrt{R / g}$, and linearising around the rest state, we have [1]

$$
\begin{aligned}
& \frac{\partial \hat{u}_{r}}{\partial r}+\frac{\hat{u}_{r}}{r}+\frac{1}{r} \frac{\partial \hat{u}_{\theta}}{\partial \theta}+\frac{\partial \hat{u}_{z}}{\partial z}=0, \\
& \hat{\mathbf{u}}_{t}=-\nabla \hat{p}+\frac{1}{\operatorname{Re}} \nabla^{2} \hat{\mathbf{u}}, \\
& \hat{u}_{r}(r=1, \theta, z, t)=\hat{u}_{\theta}(r=1, \theta, z, t)=\hat{u}_{z}(r=1, \theta, z, t)=0, \\
& \hat{u}_{r}(r, \theta, z=-h, t)=\hat{u}_{\theta}(r, \theta, z=-h, t)=\hat{u}_{z}(r, \theta, z=-h, t)=0 \\
& \hat{u}_{r}=\hat{u}_{\theta}=0, \hat{\eta}_{t}=\hat{u}_{z}, \text { on } z=0, \\
& \hat{p}-\frac{2}{\operatorname{Re}} \frac{\partial \hat{u}_{z}}{\partial z}-\hat{\eta}+\tau\left[\frac{\partial^{2} \hat{\eta}}{\partial r^{2}}+\frac{1}{r} \frac{\partial \hat{\eta}}{\partial r}+\frac{1}{r^{2}} \frac{\partial^{2} \hat{\eta}}{\partial \theta^{2}}\right]=\zeta \frac{\partial^{2} \hat{\eta}}{\partial t^{2}} \quad \text { on } z=0, \\
& \hat{\eta}(r=1, \theta, t)=0 \text { and } \int_{0}^{2 \pi} \int_{0}^{1} r \hat{\eta}(r, \theta, t) \mathrm{d} r=0 .
\end{aligned}
$$


Fig. 1 Geometry for hydroelastic oscillations of an elastic membrane covering the free surface in a circular cylinder. The liquid is of depth $h$

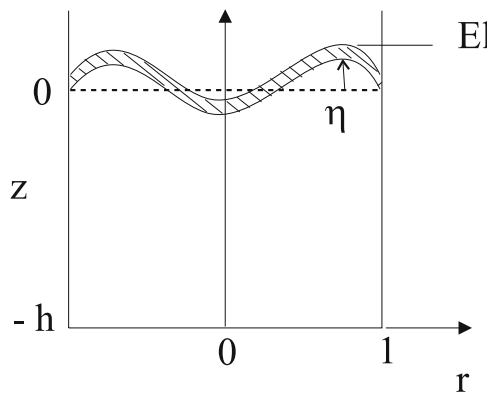

Elastic membrane

Here $\hat{u}_{r}, \hat{u}_{\theta}$ and $\hat{u}_{z}$ are the $r$-, $\theta$ - and $z$-components of velocity, $\hat{p}$ is the reduced pressure (with gravity incorporated) and $\hat{\eta}$ the deflection of the elastic membrane. $\operatorname{Re}=\sqrt{g R^{3}} / \nu$ is the gravitational Reynolds number, $\tau=T / \rho g R^{2}$ is the tension parameter and $\zeta=\xi / \rho R$ is the density ratio; $\rho$ and $\nu$ are the density and kinematic viscosity of the fluid, $T$ and $\xi$ are the tension and mass per unit area of the membrane and $g$ is the acceleration due to gravity. Note that the gravitational parameter $g^{*}$ and the tension parameter $T^{*}[1]$ equal $\operatorname{Re}^{2}$ and $\operatorname{Re}^{2} \tau$, respectively. The continuity equation is given by 1(a), the momentum equations by 1(b), the zero-velocity boundary conditions on the side wall by $1(\mathrm{c})$, the boundary condition on the bottom wall by $1(\mathrm{~d})$, the kinematic conditions on the elastic membrane by $1(\mathrm{e})$ and the deformation of the elastic membrane by $1(\mathrm{f})$. Note that, under linearisation, $1(\mathrm{e}, \mathrm{f})$ are applied on $z=0$. The fixed boundary condition for the membrane is given by $1(\mathrm{~g})$ and the volume conservation condition by $1(\mathrm{~h})$.

Anticipating the existence of axisymmetric modes, it may be noted that similar equations hold with the obvious change that there is no azimuthal dependence and that the velocity field is two-dimensional.

\section{Solution}

Since we are interested in oscillatory solutions to the system $1(\mathrm{a}-\mathrm{h})$, we seek

$\hat{u}_{r}(r, \theta, z, t)=\mathrm{e}^{\Omega t} u_{r}(r, \theta, z), \quad \hat{u}_{\theta}(r, \theta, z, t)=\mathrm{e}^{\Omega t} u_{\theta}(r, \theta, z)$,

$\hat{u}_{z}(r, \theta, z, t)=\mathrm{e}^{\Omega t} u_{z}(r, \theta, z), \quad \hat{p}(r, \theta, z, t)=\mathrm{e}^{\Omega t} p(r, \theta, z), \quad$ and $\hat{\eta}(r, \theta, t)=\mathrm{e}^{\Omega t} \eta(r, \theta)$.

where $\Omega$ is the (possibly complex) oscillatory frequency to be determined. The real and imaginary parts of $\Omega, \Omega_{r}$ abd $\Omega_{i}$ give the damping rate and frequency. Using $2(\mathrm{a}-\mathrm{e})$ in system (1), we have

$\frac{\partial u_{r}}{\partial r}+\frac{u_{r}}{r}+\frac{1}{r} \frac{\partial u_{\theta}}{\partial \theta}+\frac{\partial u_{z}}{\partial z}=0$

$\Omega \mathbf{u}=-\nabla p+\frac{1}{\operatorname{Re}} \nabla^{2} \mathbf{u}$,

$u_{r}(r=1, \theta, z)=u_{\theta}(r=1, \theta, z)=u_{z}(r=1, \theta, z)=0$,

$u_{r}(r, \theta, z=-h)=u_{\theta}(r, \theta, z=-h)=u_{z}(r, \theta, z=-h)=0$,

$u_{r}=u_{\theta}=0, \Omega \eta=u_{z}$ on $z=0$,

$p-\frac{2}{\operatorname{Re}} \frac{\partial u_{z}}{\partial z}-\eta+\tau\left[\frac{\partial^{2} \eta}{\partial r^{2}}+\frac{1}{r} \frac{\partial \eta}{\partial r}+\frac{1}{r^{2}} \frac{\partial^{2} \eta}{\partial \theta^{2}}\right]=\Omega^{2} \zeta \eta$ on $z=0$,

$\eta(r=1, \theta)=0 \quad$ and $\quad \int_{0}^{2 \pi} \int_{0}^{1} r \eta(r, \theta) \mathrm{d} r=0$.

Following Kidambi [5], we seek

$\mathbf{u}=\nabla \phi+\nabla \times \mathbf{A}, p=-\Omega \phi$, 
where $\mathbf{B}=\nabla \times \mathbf{A}$ satisfies the vector Helmholtz equation, $\nabla^{2} \mathbf{B}=\Omega \operatorname{Re} \mathbf{B}$, while $\phi$ satisfies $\nabla^{2} \phi=0$. It is easily checked that the above velocity and pressure fields, with $\mathbf{B}$ and $\phi$ as defined satisfy the continuity and the linearised Navier-Stokes equations 3(a,b).

Using the results in [10], we may write two independent solenoidal vector fields $\mathbf{B}_{\mathbf{1}}$ and $\mathbf{B}_{\mathbf{2}}$ satisfying the vector Helmholtz equation as

$$
\begin{aligned}
\mathbf{B}_{1}= & \mathrm{e}^{k z}\left[\mathbf{e}_{r}\left(\begin{array}{l}
\cos m \theta \\
-\sin m \theta
\end{array}\right)\left\{J_{m-1}(\alpha r)+J_{m+1}(\alpha r)\right\}-\mathbf{e}_{\theta}\left(\begin{array}{c}
\sin m \theta \\
\cos m \theta
\end{array}\right)\left\{J_{m-1}(\alpha r)-J_{m+1}(\alpha r)\right\}\right], \\
\mathbf{B}_{2}= & \mathrm{e}^{k z}\left[\mathbf{e}_{r} k\left(\begin{array}{c}
\cos m \theta \\
-\sin m \theta
\end{array}\right)\left\{J_{m-1}(\alpha r)-J_{m+1}(\alpha r)\right\}-\mathbf{e}_{\theta} k\left(\begin{array}{c}
\sin m \theta \\
\cos m \theta
\end{array}\right)\left\{J_{m-1}(\alpha r)+J_{m+1}(\alpha r)\right\}\right. \\
& \left.+\mathbf{e}_{z} 2 \alpha\left(\begin{array}{c}
\cos m \theta \\
\sin m \theta
\end{array}\right) J_{m}(\alpha r)\right] .
\end{aligned}
$$

Here, $\left(\mathbf{e}_{r}, \mathbf{e}_{\theta}, \mathbf{e}_{z}\right)$ are the unit vectors in the cylindrical coordinate system, $J_{m}$ is the Bessel function of the first kind of order $m$, and $\alpha=\sqrt{k^{2}-\Omega \operatorname{Re}} . m$ and $k$ are the azimuthal and axial wavenumbers. Note that out of the four possible vector fields given above, two generate radial and axial velocity fields that are symmetric about $\theta=0$ and the other two antisymmetric. We shall call these 'symmetric' and 'antisymmetric' modes, respectively, as these correspond to symmetric and antisymmetric free surface oscillations. However, the frequency of the mode with azimuthal wave number $m$ is the same, irrespective of whether it is symmetric or antisymmetric. From now on, for definiteness, we consider only symmetric modes. The scalar field $\phi$ can be immediately written down as

$\phi(r, \theta, z)=\mathrm{e}^{k z}\left(\begin{array}{c}\cos m \theta \\ \sin m \theta\end{array}\right) J_{m}(k r)$.

We now write down the velocity fields for the symmetric modes, with obvious changes for the antisymmetric ones. Anticipating imaginary eigenvalues, we scale the Bessel functions by an exponential factor $\exp \left(k_{i}\right), k_{i}=\mathfrak{I m}(k)$ to avoid the large numbers that result for Bessel functions of arguments with large imaginary parts. Thus, in what follows, $J_{m}(k r)$ actually means $J_{m}(k r) / \exp \left(k_{i}\right)$. By combining the fields $\nabla \phi, \mathbf{B}_{\mathbf{1}}$ and $\mathbf{B}_{\mathbf{2}}$ in the ratio 1:a:b, a candidate velocity field can be written as

$$
\begin{aligned}
& v_{r}(r, \theta, z)=\mathrm{e}^{k z} \cos m \theta\left[\frac{\mathrm{d}}{\mathrm{d} r} J_{m}(k r)+a\left\{J_{m-1}(\alpha r)+J_{m+1}(\alpha r)\right\}+b k\left\{J_{m-1}(\alpha r)-J_{m+1}(\alpha r)\right\}\right], \\
& v_{\theta}(r, \theta, z)=-\mathrm{e}^{k z} \sin m \theta\left[\frac{m}{r} J_{m}(k r)+a\left\{J_{m-1}(\alpha r)-J_{m+1}(\alpha r)\right\}+b k\left\{J_{m-1}(\alpha r)+J_{m+1}(\alpha r)\right\}\right], \\
& v_{z}(r, \theta, z)=\mathrm{e}^{k z} \cos m \theta\left[k J_{m}(k r)+b 2 \alpha J_{m}(\alpha r)\right] .
\end{aligned}
$$

These velocity fields define modes that we denote by $(m, n) ; m$ denotes the number of azimuthal nodes (nodal diameters) while $n$ denotes the number of nodes in the radial direction (nodal circles), excluding the one enforced by the pinned contact line at $r=1$. Thus, the first axisymmetric mode is $(0,1)$, while the first non-axisymmetric modes are $(m, 0)$. The vanishing of the velocity on the sidewall $r=1$ leads to the following system of linear equations:

$\frac{k}{2}\left\{J_{m-1}(k)-J_{m+1}(k)\right\}+a\left\{J_{m-1}(\alpha)+J_{m+1}(\alpha)\right\}+b k\left\{J_{m-1}(\alpha)-J_{m+1}(\alpha)\right\}=0$,

$m J_{m}(k)+a\left\{J_{m-1}(\alpha)-J_{m+1}(\alpha)\right\}+b k\left\{J_{m-1}(\alpha)+J_{m+1}(\alpha)\right\}=0$,

$k J_{m}(k)+b 2 \alpha J_{m}(\alpha)=0$.

For (7) to have a non-trivial solution, we need

$$
\left|\begin{array}{lll}
\frac{k}{2}\left\{J_{m-1}(k)-J_{m+1}(k)\right\} & J_{m-1}(\alpha)+J_{m+1}(\alpha) & k\left\{J_{m-1}(\alpha)-J_{m+1}(\alpha)\right\} \\
m J_{m}(k) & J_{m-1}(\alpha)-J_{m+1}(\alpha) & k\left\{J_{m-1}(\alpha)+J_{m+1}(\alpha)\right\} \\
k J_{m}(k) & 0 & 2 \alpha J_{m}(\alpha)
\end{array}\right|=0,
$$

which, on simplification, leads to an eigenvalue relation for $k$ which reads

$4 k^{2} J_{m-1}(\alpha) J_{m}(k) J_{m+1}(\alpha)+k \alpha J_{m}(\alpha)\left[J_{m-1}(k)-J_{m+1}(k)\right]\left[J_{m-1}(\alpha)-J_{m+1}(\alpha)\right]-4 m^{2} J_{m}(k) J_{m}^{2}(\alpha)=0$. 
For $k^{\star}$ and $\alpha^{\star}$ satisfying (8), Eqs. 6 will yield vector eigenfunctions provided the constants $a$ and $b$ are given by

$a=\frac{m J_{m}\left(k^{\star}\right)\left(1-k^{\star 2} / \alpha^{\star 2}\right)}{J_{m+1}\left(\alpha^{\star}\right)-J_{m-1}\left(\alpha^{\star}\right)}, \quad b=-\frac{k^{\star}}{2 \alpha^{\star}} \frac{J_{m}\left(k^{\star}\right)}{J_{m}\left(\alpha^{\star}\right)}$.

For $m \neq 0$, it is known from [5] that (8) has three sets of complex eigenvalues which we denote $\left\{\lambda_{n}\right\},\left\{\mu_{n}\right\}$ and $\left\{v_{n}\right\}$. However, the equation in [5] is simpler than the present one because (8) involves the unknown $\Omega$ which has to be determined so as to satisfy an eigenvalue problem that we will derive in the next section.

We now sketch the solution for the axisymmetric modes $(m=0)$, which is slightly different. For these modes, $\mathbf{B}_{2}(5(b))$ is the only non-zero velocity field and is given by

$\mathbf{B}_{2}=\mathrm{e}^{k z}\left[-\mathbf{e}_{r} k J_{1}(\alpha r)+\mathbf{e}_{\mathbf{z}} \alpha J_{0}(\alpha r)\right]$.

A candidate velocity field can be written as

$v_{r}(r, z)=-k \mathrm{e}^{k z}\left[J_{1}(k r)+2 b J_{1}(\alpha r)\right]$,

$v_{z}(r, z)=\mathrm{e}^{k z}\left[k J_{0}(k r)+b 2 \alpha J_{0}(\alpha r)\right]$,

where the wave number $k$ is a solution of the eigenvalue relation

$\alpha J_{1}(k) J_{0}(\alpha)-k J_{0}(k) J_{1}(\alpha)=0, \quad$ and $\quad b=-\frac{k}{2 \alpha} \frac{J_{0}(k)}{J_{0}(\alpha)}$.

The eigenvalue equation has only two sets of complex eigenvalues $\left\{\lambda_{n}\right\}$ and $\left\{\mu_{n}\right\}$.

We now formulate an eigenvalue problem for $\Omega$. Equations 3(e,f) involve $\eta(r, \theta)$ which we seek as

$\eta(r, \theta)=\cos m \theta \sum_{n=1}^{N} g_{n} J_{m}\left(\delta_{n} r\right)$,

where the $g_{n}$ are coefficients to be determined by satisfying 3(e,f). Here the $\delta_{n}$ are the zeros of $J_{m}$. Note that (9) satisfies the fixed boundary condition $3(\mathrm{~g})$ and the volume conservation condition $3(\mathrm{~h})$ for non-axisymmetric ( $m=0$ ) modes. We next write the three components of the velocity field $u_{r}, u_{\theta}$ and $u_{z}$ as linear combinations of the vector eigenfunctions given in (6). Denoting

$$
\begin{aligned}
& p_{n}^{1}(r)=\left[\frac{\mathrm{d}}{\mathrm{d} r} J_{m}\left(\lambda_{n} r\right)+a\left\{J_{m-1}\left(\alpha_{n}^{1} r\right)+J_{m+1}\left(\alpha_{n}^{1} r\right)\right\}+b \lambda_{n}\left\{J_{m-1}\left(\alpha_{n}^{1} r\right)-J_{m+1}\left(\alpha_{n}^{1} r\right)\right\}\right], \\
& p_{n}^{2}(r)=\left[\frac{m}{r} J_{m}\left(\lambda_{n} r\right)+a\left\{J_{m-1}\left(\alpha_{n}^{1} r\right)-J_{m+1}\left(\alpha_{n}^{1} r\right)\right\}+b \lambda_{n}\left\{J_{m-1}\left(\alpha_{n}^{1} r\right)+J_{m+1}\left(\alpha_{n}^{1} r\right)\right\}\right], \\
& p_{n}^{3}(r)=\left[\lambda_{n} J_{m}\left(\lambda_{n} r\right)+2 b \alpha_{n}^{1} J_{m}\left(\alpha_{n}^{1} r\right)\right], \\
& q_{n}^{1}(r)=\left[\frac{\mathrm{d}}{\mathrm{d} r} J_{m}\left(\mu_{n} r\right)+a\left\{J_{m-1}\left(\alpha_{n}^{2} r\right)+J_{m+1}\left(\alpha_{n}^{2} r\right)\right\}+b \mu_{n}\left\{J_{m-1}\left(\alpha_{n}^{2} r\right)-J_{m+1}\left(\alpha_{n}^{2} r\right)\right\}\right], \\
& q_{n}^{2}(r)=\left[\frac{m}{r} J_{m}\left(\mu_{n} r\right)+a\left\{J_{m-1}\left(\alpha_{n}^{2} r\right)-J_{m+1}\left(\alpha_{n}^{2} r\right)\right\}+b \mu_{n}\left\{J_{m-1}\left(\alpha_{n}^{2} r\right)+J_{m+1}\left(\alpha_{n}^{2} r\right)\right\}\right], \\
& q_{n}^{3}(r)=\left[\mu_{n} J_{m}\left(\mu_{n} r\right)+2 b \alpha_{n}^{2} J_{m}\left(\alpha_{n}^{2} r\right)\right], \\
& s_{n}^{1}(r)=\left[\frac{\mathrm{d}}{\mathrm{d} r} J_{m}\left(v_{n} r\right)+a\left\{J_{m-1}\left(\alpha_{n}^{3} r\right)+J_{m+1}\left(\alpha_{n}^{3} r\right)\right\}+b v_{n}\left\{J_{m-1}\left(\alpha_{n}^{3} r\right)-J_{m+1}\left(\alpha_{n}^{3} r\right)\right\}\right], \\
& s_{n}^{2}(r)=\left[\frac{m}{r} J_{m}\left(v_{n} r\right)+a\left\{J_{m-1}\left(\alpha_{n}^{3} r\right)-J_{m+1}\left(\alpha_{n}^{3} r\right)\right\}+b v_{n}\left\{J_{m-1}\left(\alpha_{n}^{3} r\right)+J_{m+1}\left(\alpha_{n}^{3} r\right)\right\}\right], \\
& s_{n}^{3}(r)=\left[v_{n} J_{m}\left(v_{n} r\right)+2 b \alpha_{n}^{3} J_{m}\left(\alpha_{n}^{3} r\right)\right],
\end{aligned}
$$


we can write the three components of the velocity field for the $m$ th azimuthal mode as

$$
\begin{aligned}
& u_{r}(r, \theta, z)=\cos m \theta \sum_{n=1}^{\infty}\left[p_{n}^{1}(r)\left(a_{n} \frac{\cosh \lambda_{n}(z+h / 2)}{\cosh \lambda_{n} \frac{h}{2}}+d_{n} \frac{\sinh \lambda_{n}(z+h / 2)}{\sinh \lambda_{n} \frac{h}{2}}\right)+q_{n}^{1}(r)\left(b_{n} \frac{\cosh \mu_{n}(z+h / 2)}{\cosh \mu_{n} \frac{h}{2}}\right.\right. \\
& \left.\left.+e_{n} \frac{\sinh \mu_{n}(z+h / 2)}{\sinh \mu_{n} \frac{h}{2}}\right)+s_{n}^{1}(r)\left(c_{n} \frac{\cosh v_{n}(z+h / 2)}{\cosh v_{n} \frac{h}{2}}+f_{n} \frac{\sinh v_{n}(z+h / 2)}{\sinh v_{n} \frac{h}{2}}\right)\right] \text {, } \\
& u_{\theta}(r, \theta, z)=-\sin m \theta \sum_{n=1}^{\infty}\left[p_{n}^{2}(r)\left(a_{n} \frac{\cosh \lambda_{n}(z+h / 2)}{\cosh \lambda_{n} \frac{h}{2}}+d_{n} \frac{\sinh \lambda_{n}(z+h / 2)}{\sinh \lambda_{n} \frac{h}{2}}\right)+q_{n}^{2}(r)\left(b_{n} \frac{\cosh \mu_{n}(z+h / 2)}{\cosh \mu_{n} \frac{h}{2}}\right.\right. \\
& \left.\left.+e_{n} \frac{\sinh \mu_{n}(z+h / 2)}{\sinh \mu_{n} \frac{h}{2}}\right)+s_{n}^{2}(r)\left(c_{n} \frac{\cosh v_{n}(z+h / 2)}{\cosh v_{n} \frac{h}{2}}+f_{n} \frac{\sinh v_{n}(z+h / 2)}{\sinh v_{n} \frac{h}{2}}\right)\right] \text {, } \\
& u_{z}(r, \theta, z)=\cos m \theta \sum_{n=1}^{\infty}\left[p_{n}^{3}(r)\left(a_{n} \frac{\sinh \lambda_{n}(z+h / 2)}{\cosh \lambda_{n} \frac{h}{2}}+d_{n} \frac{\cosh \lambda_{n}(z+h / 2)}{\sinh \lambda_{n} \frac{h}{2}}\right)+q_{n}^{3}(r)\left(b_{n} \frac{\sinh \mu_{n}(z+h / 2)}{\cosh \mu_{n} \frac{h}{2}}\right.\right. \\
& \left.\left.+e_{n} \frac{\cosh \mu_{n}(z+h / 2)}{\sinh \mu_{n} \frac{h}{2}}\right)+s_{n}^{3}(r)\left(c_{n} \frac{\sinh v_{n}(z+h / 2)}{\cosh v_{n} \frac{h}{2}}+f_{n} \frac{\cosh v_{n}(z+h / 2)}{\sinh v_{n} \frac{h}{2}}\right)\right] \text {. }
\end{aligned}
$$

In the above, $\alpha_{n}^{1}=\sqrt{\lambda_{n}^{2}-\Omega \operatorname{Re}}, \alpha_{n}^{2}=\sqrt{\mu_{n}^{2}-\Omega \operatorname{Re}}, \alpha_{n}^{3}=\sqrt{v_{n}^{2}-\Omega \operatorname{Re}}$ (these appear in $p_{n}^{j}, q_{n}^{j}$ and $s_{n}^{j}, j=$ $1,2,3) ; a_{n}, b_{n}, c_{n}, d_{n}, e_{n}, f_{n}$ are the unknown complex coefficients that have to be determined such that the remaining boundary conditions $3(\mathrm{~d}, \mathrm{e}, \mathrm{f})$, two of which also contain the unknown frequency $\Omega$, are satisfied. Note that the coefficients of $a_{n}, b_{n}, c_{n}, d_{n}, e_{n}$ and $f_{n}$ are divided by the hyperbolic functions to keep them $O(1)$ at $z=0$ and $z=-h$ where the boundary conditions will be applied. We follow a weighted-residual-method approach and require the inner product of equations $3(\mathrm{~d}, \mathrm{e}, \mathrm{f})$ with the first $N$ members of a complete set of test functions to vanish. We choose the test functions as the set $\left\{J_{m}\left(\delta_{l} r\right), l=1,2, \ldots\right\}$, where the $\delta_{l}$ are the zeros of $J_{m}$. First we project the bottom boundary conditions 3(d) and the first two of 3(e) to get the set of $5 \mathrm{~N}$ equations

$\sum_{n=1}^{N}\left(a_{n} \beta_{n l}^{i}+b_{n} \xi_{n l}^{i}+c_{n} \chi_{n l}^{i}+d_{n} \gamma_{n l}^{i}+e_{n} \rho_{n l}^{i}+f_{n} \psi_{n l}^{i}\right)=0, \quad i=1, \ldots, 5 ; \quad l=1, \ldots, N$

with the integrals $\beta_{n l}^{i}, \ldots$ defined in Appendix. Equation 12 can be written in matrix form as

$$
\mathbf{U}\left(\begin{array}{l}
\mathbf{b} \\
\mathbf{c} \\
\mathbf{d} \\
\mathbf{e} \\
\mathbf{f}
\end{array}\right)=-\mathbf{V} \mathbf{a}
$$

where $\mathbf{U}$ and $\mathbf{V}$ are defined by

$$
\mathbf{U}=\left(\begin{array}{lllll}
\mathbf{Y}_{1} & \mathbf{Z}_{1} & \mathbf{X}^{1} & \mathbf{Y}^{1} & \mathbf{Z}^{1} \\
\mathbf{Y}_{2} & \mathbf{Z}_{2} & \mathbf{X}^{2} & \mathbf{Y}^{2} & \mathbf{Z}^{2} \\
\mathbf{Y}_{3} & \mathbf{Z}_{3} & \mathbf{X}^{3} & \mathbf{Y}^{3} & \mathbf{Z}^{3} \\
\mathbf{Y}_{4} & \mathbf{Z}_{4} & \mathbf{X}^{4} & \mathbf{Y}^{4} & \mathbf{Z}^{4} \\
\mathbf{Y}_{5} & \mathbf{Z}_{5} & \mathbf{X}^{5} & \mathbf{Y}^{5} & \mathbf{Z}^{5}
\end{array}\right) \text { and } \mathbf{V}=\left(\begin{array}{l}
\mathbf{X}_{1} \\
\mathbf{X}_{2} \\
\mathbf{X}_{3} \\
\mathbf{X}_{4} \\
\mathbf{X}_{5}
\end{array}\right)
$$

An inversion of $\mathbf{U}$ gives $\mathbf{b}, \mathbf{c}, \mathbf{d}, \mathbf{e}$ and $\mathbf{f}$ in terms of $\mathbf{a}$. Note that $\mathbf{U}$ and $\mathbf{V}$ have dimensions $5 N \times 5 N$ and $5 N \times N$. 
Expressing the pressure in terms of the potential by 4(b) and projecting the last equation of 3(e) and 3(f) onto the set $\left\{J_{m}\left(\delta_{l} r\right)\right\}$, we get $2 N$ equations:

$$
\begin{aligned}
& \sum_{n=1}^{N}\left(a_{n} \beta_{n l}^{6}+b_{n} \xi_{n l}^{6}+c_{n} \chi_{n l}^{6}+d_{n} \gamma_{n l}^{6}+e_{n} \rho_{n l}^{6}+f_{n} \psi_{n l}^{6}\right)=\Omega \sum_{n=1}^{N} g_{n} \theta_{n l}^{6}, \quad l=1, \ldots N, \\
& \zeta \Omega^{2} \sum_{n=1}^{N} g_{n} \theta_{n l}^{6}+\Omega \sum_{n=1}^{N}\left(a_{n} \beta_{n l}^{7}+b_{n} \xi_{n l}^{7}+c_{n} \chi_{n l}^{7}+d_{n} \gamma_{n l}^{7}+e_{n} \rho_{n l}^{7}+f_{n} \psi_{n l}^{7}\right) \\
& \quad+\sum_{n=1}^{N}\left(a_{n} \beta_{n l}^{8}+b_{n} \xi_{n l}^{8}+c_{n} \chi_{n l}^{8}+d_{n} \gamma_{n l}^{8}+e_{n} \rho_{n l}^{8}+f_{n} \psi_{n l}^{8}+g_{n} \theta_{n l}^{8}\right)=0, \quad l=1, \ldots N .
\end{aligned}
$$

The integrals appearing in (14) are also given in Appendix. We use (13) to write (14) in terms of only $\mathbf{a}$ and $\mathbf{g}$ as $\mathbf{C}_{1} \mathbf{a}=\Omega \mathbf{D}_{1} \mathbf{g}, \quad \Omega^{2} \mathbf{D}_{2} \mathbf{g}+\Omega \mathbf{C}_{2} \mathbf{a}+\mathbf{C}_{3} \mathbf{a}+\mathbf{D}_{3} \mathbf{g}=0$.

Using 15(a) to express $\mathbf{a}$ in terms of $\mathbf{g}$, we can finally write 15 (b) as the nonlinear eigenvalue problem

$\left(\mathbf{E}_{1}(\Omega ; \operatorname{Re}, \tau, \zeta) \Omega^{2}+\mathbf{E}_{2}(\Omega ; \operatorname{Re}, \tau, \zeta) \Omega+\mathbf{E}_{3}(\Omega ; \operatorname{Re}, \tau, \zeta)\right) \mathbf{g}=0$

where $\mathbf{E}_{1}, \mathbf{E}_{2}$ and $\mathbf{E}_{3}$ are $N \times N$ matrices with

$\mathbf{E}_{1}=\mathbf{D}_{2}+\mathbf{C}_{2} \mathbf{C}_{1}^{-1} \mathbf{D}_{1}, \quad \mathbf{E}_{2}=\mathbf{C}_{3} \mathbf{C}_{1}{ }^{-1} \mathbf{D}_{1}$ and $\quad \mathbf{E}_{3}=\mathbf{D}_{3}$.

Note that, although (16) has the formal appearance of a generalised nonlinear eigenvalue problem, its solution requires iteration as the matrices $\mathbf{E}_{1}, \mathbf{E}_{2}$ and $\mathbf{E}_{3}$ are functions of $\Omega$. The numerical procedure consists of the following steps -

1. Start with an initial guess for $\Omega, \Omega_{i}$.

2. For this value of $\Omega$ and the given $\mathrm{Re}$, determine the eigenvalues $\lambda_{n}, \mu_{n}$ and $v_{n}$.

3. Compute the matrices $\mathbf{E}_{\mathbf{1}}, \mathbf{E}_{2}$ and $\mathbf{E}_{3}$.

4. Solve the eigenvalue problem (16) and obtain $\Omega_{f}$.

5. If $\mathfrak{R e}\left\{\Omega_{f}-\Omega_{i}\right\}$ and $\mathfrak{I m}\left\{\Omega_{f}-\Omega_{i}\right\}<\epsilon$ for a chosen tolerance, the calculation is done. If not, repeat (1-5) with $\Omega_{i}=\alpha_{0} \Omega_{i}+\left(1-\alpha_{o}\right) \Omega_{f}$, with $\alpha_{o} \in(0,1)$. We have used $\alpha_{o}=0.5$.

The nonlinear eigenvalue problem (16) can be written as a linear eigenvalue problem of twice the dimension by introducing the vector $\mathbf{v}=\Omega \mathbf{g}$; thus the original problem can be written

$$
\left(\begin{array}{cc}
\mathbf{0} & \mathbf{1} \\
-\mathbf{E}_{1}{ }^{-1} \mathbf{E}_{3} & -\mathbf{E}_{1}^{-1} \mathbf{E}_{2}
\end{array}\right)\left(\begin{array}{l}
\mathbf{g} \\
\mathbf{v}
\end{array}\right)=\Omega\left(\begin{array}{l}
\mathbf{g} \\
\mathbf{v}
\end{array}\right)
$$

where $\mathbf{0}$ is the $N \times N$ zero matrix and $\mathbf{1}$ is the identity matrix of dimension $N$. (17) has been solved by the LAPACK routine ZGEEV. The pinned contact line frequencies for infinite depth and a flat interface have been used as starting values; these are then continued in the $h-\operatorname{Re}-\tau-\zeta$ space to the required parameter values. In general the procedure works well and convergence is achieved under ten iterations. Note that this procedure has to be repeated for the different modes, i.e., even though $N \Omega$ are obtained by solving (17), all these will not be the correct values as the iteration was performed with respect to only one of those temporal eigenvalues. In other words, each modal frequency has corresponding to it a different set of spatial eigenvalues. The axisymmetric case $m=0$ is more subtle. Now mass conservation is not automatic unlike in the non-axisymmetric case; this defines a constraint on the $g_{n}$ appearing in $\eta$ given by (9). However, using this and the fact that a constant term has to be included in the expansion for $\phi[11]$ leads to a similar eigenvalue problem as for the non-axisymmetric modes.

\section{Results and discussion}

The frequency and damping rate of a given mode depend on the four parameters $\operatorname{Re}, h, \tau$ and $\zeta$. Since the parameter space is large, we will sketch this dependence for representative values of these parameters. We start off with a 
Table 1 Convergence study for the frequency and damping rate of the $(1,0)$ mode

20 and 40 modes have been used $h=2, \tau=0.1$, $\zeta=0.01$

\begin{tabular}{|c|c|c|c|c|}
\hline \multirow[t]{2}{*}{$\mathrm{Re}$} & \multicolumn{2}{|l|}{$-\Omega_{r}$} & \multicolumn{2}{|l|}{$\Omega_{i}$} \\
\hline & $N=20$ & $N=40$ & $N=20$ & $N=40$ \\
\hline 100 & 0.1905 & 0.1907 & 2.190 & 2.190 \\
\hline 1000 & 0.0546 & 0.0546 & 2.302 & 2.303 \\
\hline 10000 & 0.0169 & 0.0165 & 2.336 & 2.338 \\
\hline
\end{tabular}

validation of the current results. A convergence study over a two-decadal range of Re is presented in Table 1; the calculations with $N=20$ and $N=40$ modes show convergence to three decimal places for both frequency and damping rate. In most of the calculations presented below, the bottom wall boundary conditions are satisfied to $O\left(10^{-10}\right)$ and the convergence criterion for the iterative procedure of Sect. $3.1 ; \epsilon$ has been fixed at $10^{-6}$.

Extensive experimental results have been published by [8] for the viscous oscillations of a free surface with pinned contact line in a circular cylinder. The governing equations 3(a-d) are the same; of the triad 3(e), only the last is valid. The first two have to be replaced by the two shear stress equations

$\frac{\partial u_{r}}{\partial z}+\frac{\partial u_{z}}{\partial r}=0, \quad \frac{\partial u_{\theta}}{\partial z}+\frac{1}{r} \frac{\partial u_{z}}{\partial \theta}=0$,

while $\zeta$ is zero in 3(f) (there is no elastic cover) and $\tau=1 /$ Bo where Bo is the Bond number and is related to the surface tension of the liquid rather than the tension of the elastic structure. The details of the calculation procedure can be found in [6]. Table 2 provides a comparison of the results obtained with the present method, the experimental results of [8] and the asymptotic calculations of Martel et al. [9]. The results with the present method are in closer agreement with the experimental values at low Re and are comparable at high Re. Forty eigenmodes have been used to obtain these results.

It is well-known that, in general, viscosity reduces the oscillation frequency of a linear damped oscillator; in particular, Martel et al. [9], Howell et al. [8], Nicolás [12] and Kidambi [13] have shown this to be true for damped surface capillary-gravity waves in a container.

Figure 2 shows the inviscid frequency $(\operatorname{Re}=\infty)$ and the frequency for $\operatorname{Re}=100$ for the $(1,0)$ mode for a range of $\zeta$; the inviscid frequency is larger. This holds true for other modes and other values of $h, \zeta$ and $\tau$ as well. The inviscid frequencies have been calculated by appropriately modifying the procedure in [11] to account for the elastic cover. The damping rate, on the other hand, is zero for $\mathrm{Re}=\infty$ and increases with decreasing Re. It is known from studies of damping of capillary-gravity waves [12,13] that for a given mode, fluid depth and Bond number, there exists a critical $\mathrm{Re}, \mathrm{Re}_{\mathrm{cr}}$ below which $\Omega_{i}=0$ and $\Omega_{r}$ becomes double-valued. Figure 3 shows the complex frequency of the lowest three modes for $\operatorname{Re} \rightarrow 0 ; h=1, \tau=0.1, \zeta=0.01$. It is clear that the modes are overdamped for $\mathrm{Re}<\mathrm{Re}_{\mathrm{cr}}$, the latter increasing with increasing mode number. Figure 4 shows similar data for a very heavy cover, with $\zeta=10$. The increase in the mass of the cover leads to $\operatorname{Re}_{\mathrm{cr}} \approx 0$. In the figures we have shown only the lower branch of $-\Omega_{r}$ for $\mathrm{Re}<\mathrm{Re}_{\mathrm{cr}}$ as this is the branch that decays the slowest. Figure 5 shows the results for a shallow depth of $h=0.3$ and $\tau=0.1$ for the $(1,0)$ mode; light $(\zeta=0.01)$ and heavy $(\zeta=10)$ covers have been considered. This figure should be compared with Figs. 3 and 4, where $h=1$. The shallow depth means a larger damping rate (about twice for $\zeta=0.01$ and ten times for the heavier cover $\zeta=10$ at $\operatorname{Re}=30$ ) which in turn implies a higher $\operatorname{Re}_{\mathrm{cr}}$. $\operatorname{Re}_{\mathrm{cr}} \approx 30$ for the shallow depth as against a value of 4 for $h=1$. This means that there exist parameter values (for example, $\operatorname{Re}<30, h=0.3, \tau=0.1$ here) for which the frequency increases with increasing $\zeta$, even though in general the opposite is true.

Another interesting question is the relation of the slosh frequency and damping-rate with an elastic cover present to those when there is no elastic cover. When there is no elastic cover, the contact line is free to move in general; it can also be arranged to be at rest as in the experiment of Howell et al. [8]. In the former case, the frequency increases if an elastic cover is present while in the latter, it decreases. Since the modelling of the viscous moving contact line is still an unresolved issue, we will consider the inviscid case for the $(1,0)$ mode with and without an 
Table 2 Comparison of theoretical predictions $\left(\Omega_{r}^{t}, \Omega_{i}^{t}\right)$ with experimental measurements $\left(\Omega_{r}^{e}, \Omega_{i}^{e}\right)$ for $h=1.379$ and Bo $=365$

\begin{tabular}{|c|c|c|c|c|c|c|c|}
\hline $\mathrm{Re}$ & $C$ & $\Omega_{r}^{t} / \Omega_{r}^{e}$ & $-\Omega_{r}^{e}$ & $\Omega_{r}^{t *} / \Omega_{r}^{e}$ & $\Omega_{i}^{t} / \Omega_{i}^{e}$ & $\Omega_{i}^{e}$ & $\Omega_{i}^{t *} / \Omega_{i}^{e}$ \\
\hline \multicolumn{8}{|l|}{$(1,0)$ mode } \\
\hline 13077.02 & $7.647 \times 10^{-5}$ & 1.049 & 0.0060 & 0.963 & 1.006 & 1.438 & 1.006 \\
\hline 2610.97 & $3.830 \times 10^{-4}$ & 0.967 & 0.0155 & 1.013 & 1.008 & 1.430 & 1.009 \\
\hline 272.48 & $3.670 \times 10^{-3}$ & 0.987 & 0.0680 & 1.197 & 1.008 & 1.405 & 1.011 \\
\hline \multicolumn{8}{|l|}{$(2,0)$ mode } \\
\hline 13077.02 & $7.647 \times 10^{-5}$ & 0.993 & 0.0090 & 0.973 & 1.007 & 1.899 & 1.006 \\
\hline 2610.97 & $3.830 \times 10^{-4}$ & 0.970 & 0.0254 & 1.035 & 1.008 & 1.889 & 1.009 \\
\hline 271.44 & $3.684 \times 10^{-3}$ & 0.944 & 0.1364 & 1.169 & 1.006 & 1.852 & 1.013 \\
\hline \multicolumn{8}{|l|}{$(3,0)$ mode } \\
\hline 13077.02 & $7.647 \times 10^{-5}$ & 1.010 & 0.0117 & 0.996 & 1.007 & 2.272 & 1.006 \\
\hline 1321.00 & $7.570 \times 10^{-4}$ & 0.965 & 0.0629 & 1.038 & 1.009 & 2.252 & 1.009 \\
\hline 568.51 & $1.759 \times 10^{-3}$ & 0.962 & 0.1166 & 1.130 & 1.007 & 2.234 & 1.012 \\
\hline \multicolumn{8}{|l|}{$(1,1)$ mode } \\
\hline 13077.02 & $7.647 \times 10^{-5}$ & 0.962 & 0.0082 & 0.948 & 1.006 & 2.501 & 1.005 \\
\hline 1318.57 & $7.584 \times 10^{-4}$ & 0.982 & 0.0557 & 1.081 & 1.004 & 2.493 & 1.007 \\
\hline 570.78 & $1.752 \times 10^{-3}$ & 0.968 & 0.1170 & 1.130 & 1.004 & 2.483 & 1.010 \\
\hline \multicolumn{8}{|l|}{$(4,0)$ mode } \\
\hline 13077.02 & $7.647 \times 10^{-5}$ & 0.965 & 0.0155 & 0.946 & 1.008 & 2.609 & 1.006 \\
\hline 6480.88 & $1.543 \times 10^{-4}$ & 0.958 & 0.0252 & 0.984 & 1.008 & 2.604 & 1.006 \\
\hline 2620.55 & $3.816 \times 10^{-4}$ & 1.013 & 0.0501 & 1.012 & 1.008 & 2.596 & 1.010 \\
\hline
\end{tabular}

The starred quantities are theoretical values from [9]. $C=1 / \mathrm{Re}$

Fig. 2 Variation of the frequency with mass parameter $\zeta$ for $h=1, \tau=0.01$. Results for $\operatorname{Re}=\infty$ (inviscid conditions) and $\operatorname{Re}=100$ are represented by the dashed and solid lines, respectively

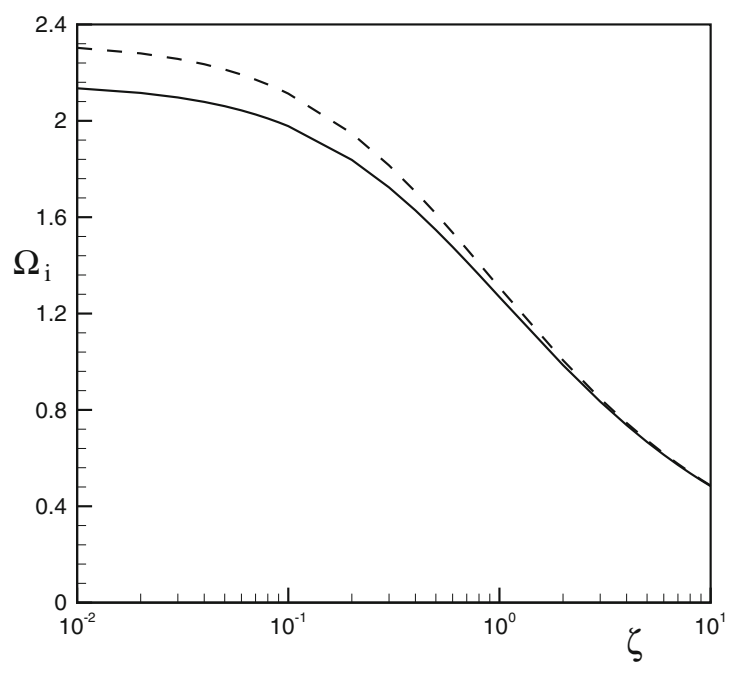

elastic cover. The frequency in the latter case, of an inviscid moving contact line, is given by the classical result [14], $\Omega_{n}^{2}=\delta_{n}\left(1+\delta_{n}^{2} /\right.$ Bo $) \tanh \delta_{n} h$, where $J_{m}^{\prime}\left(\delta_{n}\right)=0$. We compare the frequencies for the two cases in Fig. 6; the elastic cover increases the natural frequency because the interface is more constrained when a cover is present; for example, the contact line is free to move in the absence of a cover whereas it is pinned at the cylinder wall with an elastic cover present. This is the case considered in [15] where one of the conclusions is that 'the covering of a free 

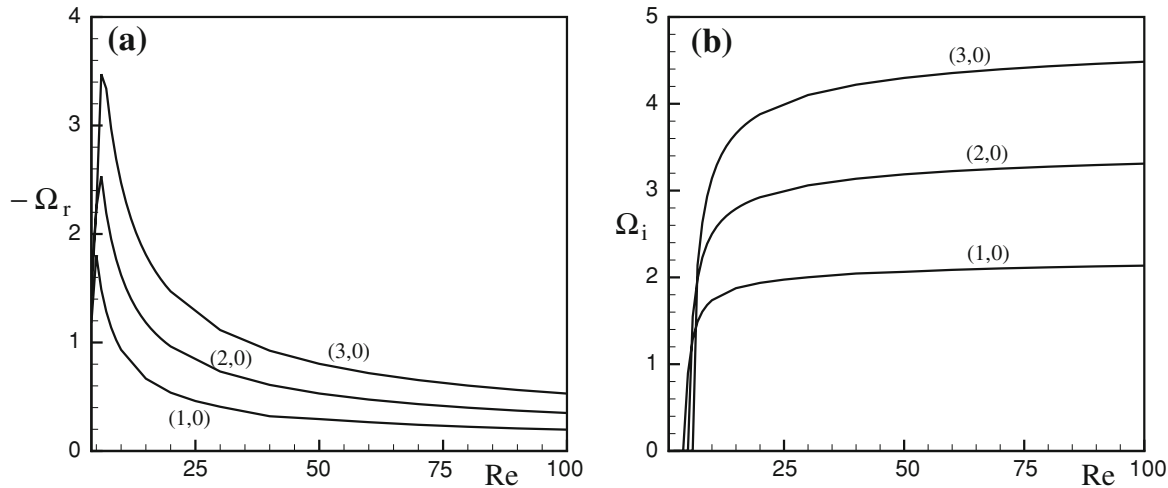

Fig. 3 Variation of the coupled (a) damping rates (b) frequencies of the lowest modes as $\operatorname{Re} \rightarrow 0 . \zeta=10^{-2}, h=1$ and $\tau=0.1$
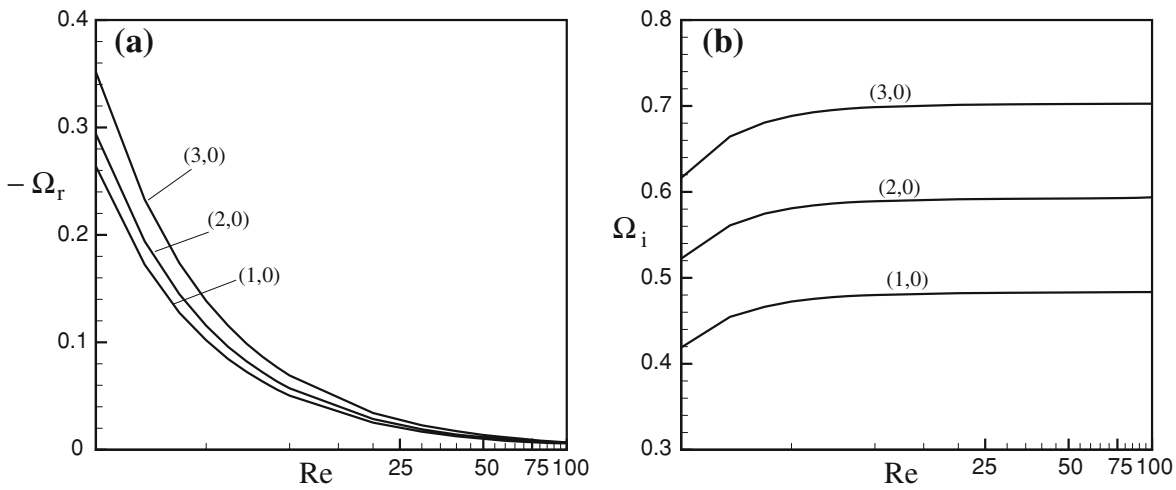

Fig. 4 Variation of the coupled (a) damping rates (b) frequencies of the lowest modes as $\operatorname{Re} \rightarrow 0 . \zeta=10, h=1$ and $\tau=0.1$
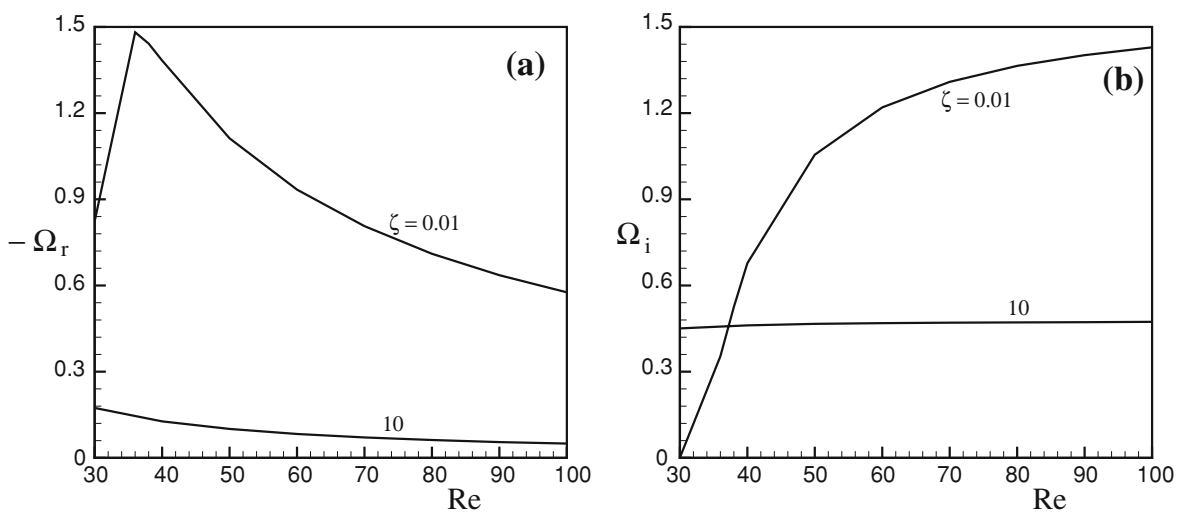

Fig. 5 Variation of the coupled (a) damping rates (b) frequencies of the $(1,0)$ mode for shallow depth $h=0.3$. There are parameter ranges where $\Omega_{i}$ is bigger for $\zeta=10$ than for $\zeta=0.01 . \tau=0.1$

liquid surface with a flexible membrane or an elastic thin plate increases the frequencies.' That it is the constraint which increases the frequency becomes clear if the case of an elastic cover is compared with the case of no cover, but with a pinned contact line.

Figure 7 shows the frequency and damping-rate variation with the tension parameter $\tau$ for the $(2,0)$ mode for $\operatorname{Re}=100, h=1$ and $\zeta=10^{-4}$ with and without an elastic cover present. Twenty modes have been used in these calculations. The frequency for the no-cover case is higher while the damping rate is larger with the cover present. The latter is easily explained as being due to the boundary layer at the elastic cover being stronger than the 
Fig. 6 Variation of the inviscid frequency with liquid depth $h$ for $\zeta=10^{-3}, \tau=0.002$

Results with and without a elastic cover are represented by the solid and dashed lines, respectively
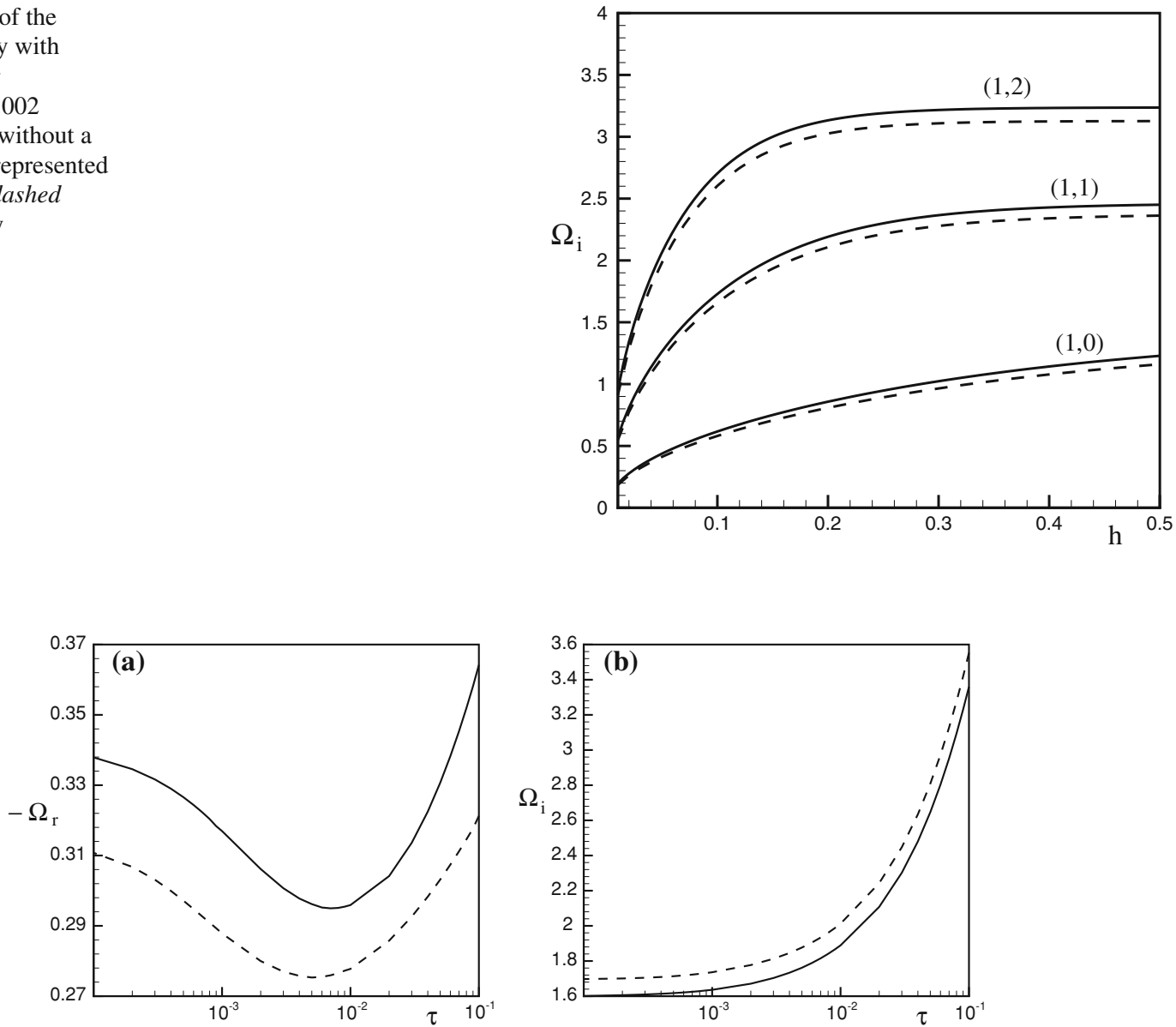

Fig. 7 Variation of the coupled (a) damping rates (b) frequencies of the (2,0) mode with tension parameter $\tau ; \zeta=10^{-4}, h=1$ and $\mathrm{Re}=100$. The solid and dashed lines show the results with and without an elastic cover present. In the latter case, the contact line is pinned

free-surface boundary layer that would obtain in the absence of a cover. The decreased frequency in the presence of a cover is more subtle and could be a viscous effect. We conjecture that the higher viscous action resulting from the presence of a cover leads to a greater reduction in the natural frequency. This conjecture can be tested by comparing the two cases for a higher Reynolds number; the frequencies should be much closer as the viscous action is much weaker in this case. Figure 8 shows this to be indeed the case.

We now explore the variation of the frequency and damping rate of the lowest slosh modes with $h, \tau$ and $\zeta$. Figure $9(a, b)$ show the variation of the damping rate and frequency of the $(1,0),(2,0),(3,0)$ and $(1,1)$ modes with liquid depth $h . \zeta=0.01$ and $\tau=0.1$, being the parameters in [1] and [3], have been used for these calculations with a view to comparing the results. The latter employ different non-dimensional parameters from ours; Table 3 gives the relationship between the two. The results in [1] are for the parameter values $g^{*}=10^{4}, T^{*}=10^{3}$ and $\mu^{*}=0.01$. These yield parameter values $\operatorname{Re}=100, \tau=0.1$ and $\zeta=0.01$ for the present study. Also, $\Omega_{b}=100 \Omega$. Figure $9(a, b)$ are to be compared with Figs. $3-6$ in $[1] .^{1}$

\footnotetext{
1 These results are assumed to be valid for $0.5 \geq h / R$; this is why the solid lines in these figures end abruptly. For the (1,1) and (3,0) modes (Figs. 4 and 6 of [1]), the results have been plotted for $h / R<0.5$; these have been obtained by a different analytic procedure, as outlined in [3]. Note that the frequency curves for $h / R<0.5$ do not continue smoothly to the ones for $h / R \geq 0.5$ (they are not even close); the damping rate behaviour is somewhat better.
} 

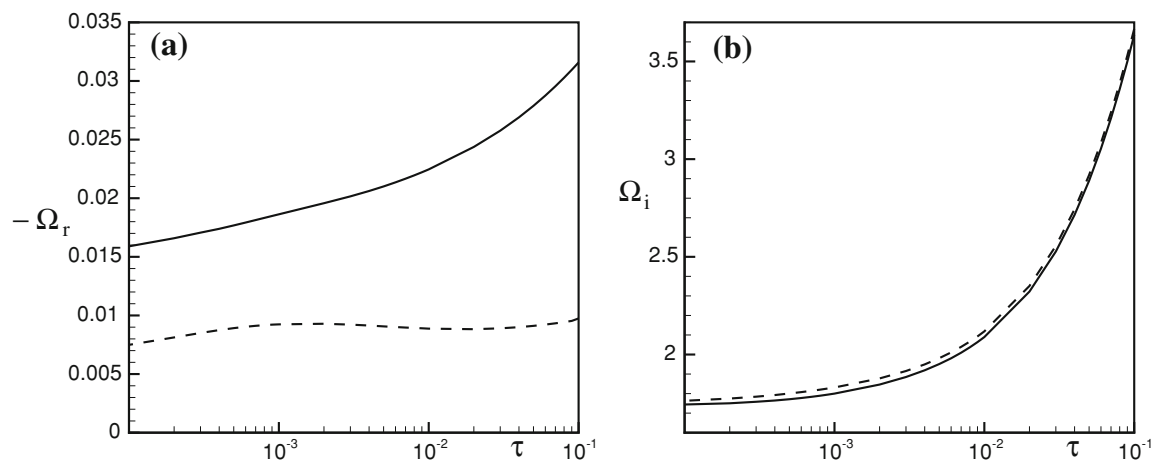

Fig. 8 Variation of the coupled (a) damping rates (b) frequencies of the (2,0) mode with tension parameter $\tau$. All parameters except $\mathrm{Re}$ are same as in Fig. 7. Re $=10000$. The solid and dashed lines show the results with and without an elastic cover present. Note that the frequencies for the two cases are much closer than in Fig. 7
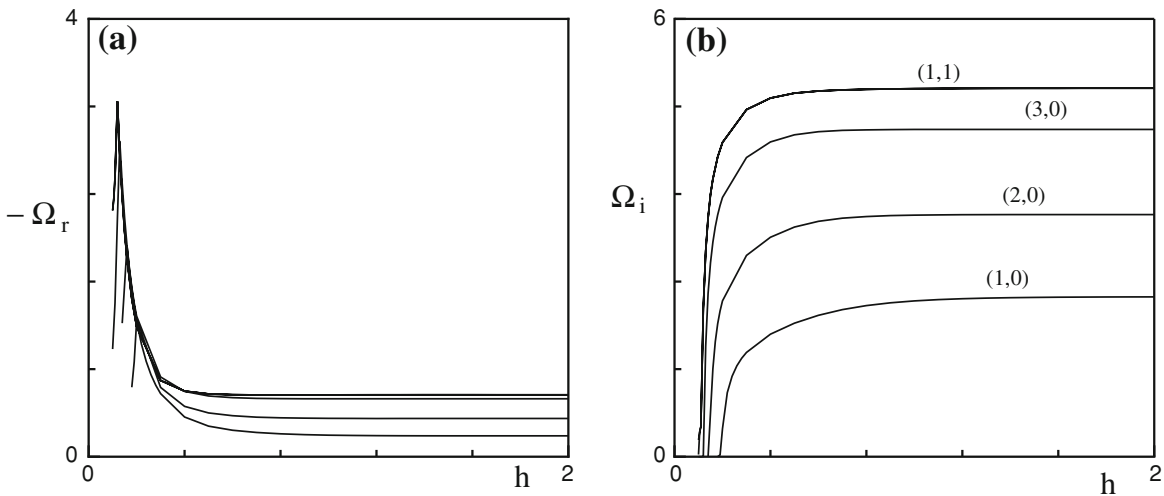

Fig. 9 Variation of the coupled (a) damping rates (b) frequencies of the $(1,0),(2,0),(3,0)$ and $(1,1)$ modes with depth. In a), the curves from top to bottom correspond to the $(1,1),(3,0),(2,0)$ and $(1,0)$ modes, respectively. $\zeta=10^{-2}, \tau=0.1$ and $\operatorname{Re}=100$

Table 3 The relation between the parameters in [1] and the present work

\begin{tabular}{ll}
\hline 1$]$ & Present \\
\hline$g^{*}=g R^{3} / \nu^{2}$ & $\operatorname{Re}=\sqrt{g R^{3}} / v=\sqrt{g^{*}}$ \\
$T^{*}=T R / \rho v^{2}$ & $\tau=T / \rho g R^{2}=T^{*} / g^{*}$ \\
$\mu^{*}=\mu / \rho R$ & $\zeta=\xi / \rho R$ \\
$\Omega_{b}=\Omega_{d} R^{2} / v$ & $\Omega=\Omega_{d} \sqrt{R / g}=\Omega_{b} / \operatorname{Re}$ \\
\hline
\end{tabular}

For a given mode, the frequencies increase with depth and a constant value is attained around a depth $h^{*}$ that is mode-dependent; $h^{*}$ is higher for the lower modes implying that the lowest modes have the largest slosh mass participating in the motion of the liquid-structure system. For fixed depth, the higher modes oscillate at higher frequencies; the rate of increase of the frequency with depth is also higher. The damping rates, for a given mode, decrease with depth due to the weaker boundary layers. For fixed depth, the higher modes have the higher damping rates; they decay faster.

The present calculations, as well as those in [1], show the coupled frequency and damping rates to be almost constant for approximately $h / R>0.5$; however, the two methods yield quite different constant values as shown in Table 4. The worst discrepancy is for the $(1,0)$ mode where the present calculation yields a damping rate that is more than four times that given in [1]. The other damping rates are also off by values ranging from $20 \%$ to 
Table 4 Comparison of damping rates and frequencies between present results and [1]

\begin{tabular}{llllll}
\cline { 2 - 4 } & $(1,0)$ & 0.191 & 0.047 & 2.19 & 2.01 \\
$\mathrm{Re}=100, h=2, \tau=$ & 0.349 & 0.135 & 3.32 & 2.98 \\
$0.1, \zeta=0.01$ & $(2,0)$ & 0.529 & 0.435 & 4.49 & 4.22 \\
\hline
\end{tabular}
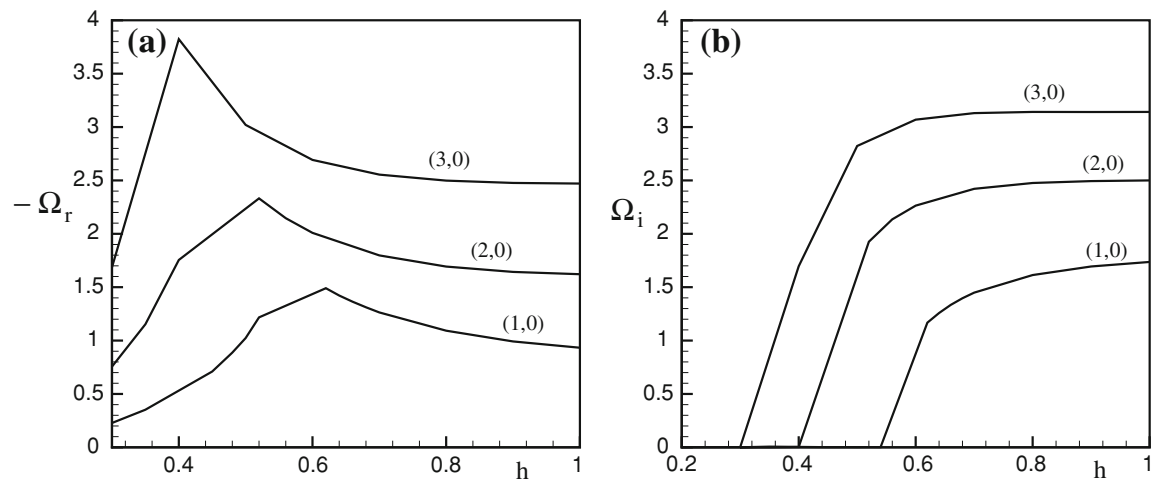

Fig. 10 Variation of the coupled (a) damping rates (b) frequencies of the $(1,0),(2,0)$ and $(3,0)$ modes with depth. $\zeta=10^{-2}, \tau=0.1$ and $\operatorname{Re}=10$

Table 5 Comparison of $h_{\mathrm{cr}}$ between present results and [3] for the lowest three modes

\begin{tabular}{|c|c|c|c|}
\hline \multirow{2}{*}{$\begin{array}{l}\text { Table } 5 \text { Comparison of } h_{\mathrm{cr}} \\
\text { between present results and } \\
\text { [3] for the lowest three } \\
\text { modes }\end{array}$} & \multirow[t]{2}{*}{ Mode } & \multicolumn{2}{|l|}{$h_{\mathrm{cr}}$} \\
\hline & & Present & [3] \\
\hline \multirow{3}{*}{$\begin{array}{l}\operatorname{Re}=100, \tau=0.1, \zeta= \\
0.01\end{array}$} & $(1,0)$ & 0.19 & 0.5 \\
\hline & $(2,0)$ & 0.14 & 0.5 \\
\hline & $(3,0)$ & 0.12 & 0.16 \\
\hline
\end{tabular}

$250 \%$ with better agreement for the higher modes. The frequencies show better agreement; the three lowest modal frequencies differ by $6-10 \%$ while the frequency of the $(1,1)$ mode is off by about $40 \%$.

From Fig. 9, we also see that, for shallow depths, the frequency (damping rate) rapidly decreases (increases) with decreasing depth; at a critical depth $h_{\mathrm{cr}}$, the frequency goes to zero and the damping rate becomes double valued with one branch continuing below the original curve, the other above. This is the familiar overdamped behaviour of a damped linear oscillator; only aperiodic slosh motions occur for $h<h_{\mathrm{cr}}$. In Fig. 9(a) we have shown only the lower branches for $h<h_{\mathrm{cr}}$. The values of $h_{\mathrm{cr}}$ are given in Table 5; corresponding values from [3] are also included. While both calculations show $h_{\mathrm{cr}}$ decreasing with increasing mode number, the actual values from the two calculations are quite different. We show similar results for the lowest three modes for a lower $\mathrm{Re}=10$ in Fig. 10 . Since the damping rates are much higher for the lower Re, (about five times at $h=1$ for the $(1,0)$ mode), aperiodic motions set in at much larger liquid depths. Thus, for instance, $h_{\mathrm{cr}} \approx 0.3,0.4$ and 0.54 for the $(3,0),(2,0)$ and $(1,0)$ modes, respectively.

We plot, in Fig. 11, the damping rate and frequency variations for a three decadal variation of the tension parameter $\tau$ for $\zeta=0.01, h=1$ and $\operatorname{Re}=100$. The frequencies are almost constant for $\tau<\tau^{*}$, where $\tau^{*}$ decreases with increasing mode number. For a given mode, the frequencies increase with increasing $\tau$ with the higher modes 

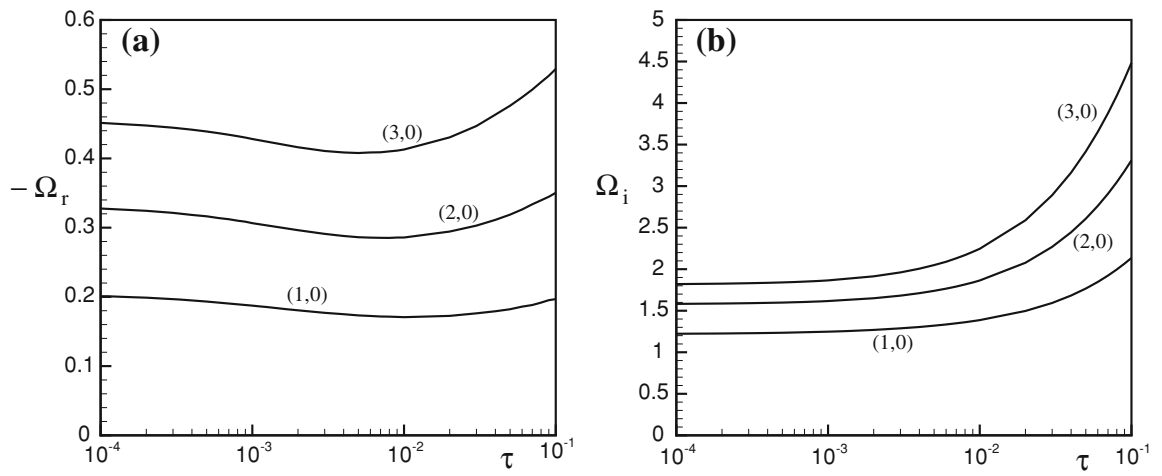

Fig. 11 Variation of the coupled (a) damping rates (b) frequencies of the (1,0), (2,0) and (3,0) modes with $\tau . \zeta=10^{-2}, h=1$ and $\operatorname{Re}=100$
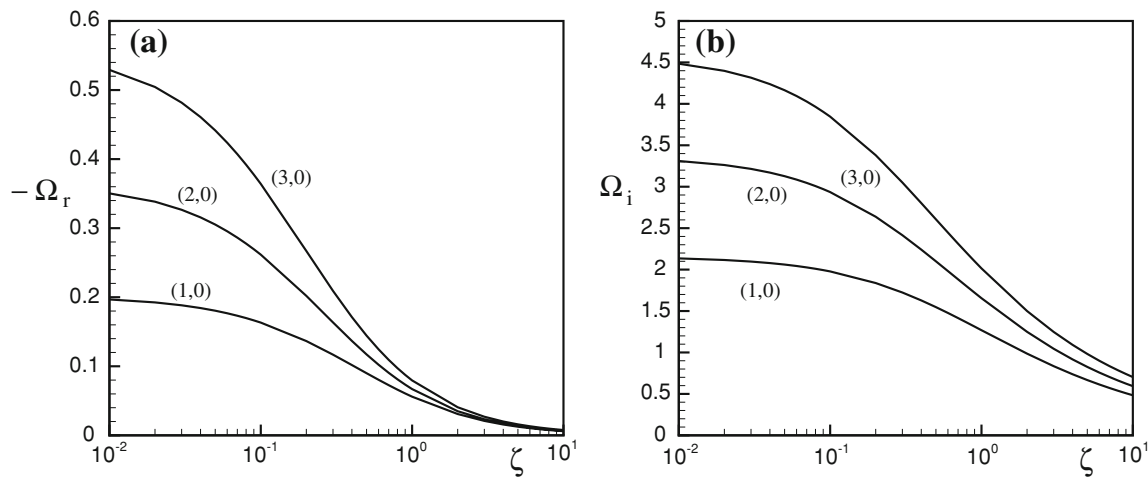

Fig. 12 Variation of the coupled (a) damping rates (b) frequencies of the $(1,0),(2,0)$ and $(3,0)$ modes with $\zeta . h=1, \tau=0.1$ and $\mathrm{Re}=100$

showing the largest increase. The damping rates show an interesting dip before again increasing for large $\tau^{*}$; these changes are however only a fraction of the mean value $(\approx 10 \%, 20 \%, 20 \%$ over a three decadal range for the lowest three modes). The increase for large $\tau$ is in accordance with the statements in [1, p. 11]. Finally, we show the variation of the frequency and the damping rate with $\zeta$ in Fig. 12. Both decrease with increasing $\zeta$; however there exist parameter ranges where $\Omega_{i}$ increases with increasing $\zeta$ as was shown earlier (Fig. 5).

\section{Conclusion}

In the introduction of [1], the authors state: 'Both solutions, i.e., the one of [29] $]^{2}$ and that presented here, are approximations to an exact solution which is not yet available. For that reason, and especially for the assumed analytical structure of these two cases the results cannot coincide and cannot exhibit at a certain $h / a$ - value complete agreement. ... We must emphasize that for these reasons we cannot expect a complete agreement in the transition zone from shallow to large aspect ratio containers.'

In this paper, we have presented an 'exact' solution that is valid for any liquid depth and there is no problem in the 'transition' zone from shallow to large depths. The use of complex eigenfunctions makes the representation rich enough to satisfy the wall and bottom boundary conditions simultaneously, besides the ones on the elastic membrane. We have presented calculations of the complex frequency for the lowest modes over a wide range of

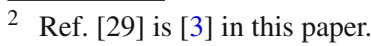


parameters: Re ranging from 1 to 10000 , depths ranging from 0.1 to $2, \tau$ ranging from 0.0001 to 0.1 and $\zeta$ ranging from 0.01 to 10 . The broad conclusions that can be drawn from these calculations are summarised below:

1. The coupled (hydroelastic) frequencies are higher when compared to the slosh frequencies of an uncovered free surface only if the contact line is free to move in the latter case. This was the case considered in [15] and is also the basis for the third conclusion in [1]. However, the hydroelastic frequencies are lower in comparison with the slosh frequencies of an uncovered free surface if the contact line is pinned. We conjecture that this is because of the increased damping rate with a cover which then results in a reduction of the frequency in comparison with the case of no cover. Support for the conjecture comes from the results that show the frequencies to be closer as $\operatorname{Re} \rightarrow \infty$.

2. For given liquid volume and membrane (fixed $h, \tau$ and $\zeta$ ), the frequencies (damping rates) increase (decrease) with decreasing liquid viscosity (increasing $\mathrm{Re}$ ). In the limit $\mathrm{Re} \rightarrow \infty$, they approach the inviscid frequency and zero, respectively. For increasing viscosity (decreasing $R e$ ), the frequencies decrease and vanish for $\operatorname{Re}<\operatorname{Re}_{c r}$. This is the well known behaviour of a damped linear oscillator.

- $R_{\text {cr }}$ increases with increasing mode number.

- $\mathrm{Re}_{\mathrm{cr}}$ increases with decreasing liquid depth.

- $\operatorname{Re}_{\mathrm{cr}}$ decreases with increasing mass parameter $\zeta$. For very heavy covers, $\operatorname{Re} \mathrm{e}_{\mathrm{cr}} \approx 0$.

3. For given liquid and membrane (fixed $\operatorname{Re}, \tau$ and $\zeta$ ), the frequencies of any mode decrease with decreasing liquid volume (decreasing depth). For a depth below the critical depth $\left(h<h_{\mathrm{cr}}\right)$, the frequencies vanish. The damping rates, on the other hand, increase with decreasing depth. At $h=h_{\mathrm{cr}}$, they become double valued with one value decreasing and the other increasing with further decrease in depth. Since the lower value controls the overall damping rate, we have followed only this branch.

- $h_{\mathrm{cr}}$ increases with decreasing mode number. This means that the range of depths for which the higher modes exhibit aperiodic decay is smaller.

- $h_{\mathrm{cr}}$ increases with decreasing Re.

4. For given liquid and membrane tension (fixed $\operatorname{Re}, h$ and $\tau$ ), the frequencies and damping rates decrease with an increasingly heavy cover (increasing $\zeta$ ). However, there exist parameter ranges (typically small volumes of highly viscous liquids) wherein the frequencies increase with increasing $\zeta$. In these ranges, the lighter cover produces overdamped oscillations while periodic oscillations are produced with the heavier cover.

5. For given liquid and membrane (fixed $h$, Re and $\zeta$ ), the frequencies increase with increasing membrane tension (increasing $\tau$ ) while the damping rates increase for large $\tau$; for smaller Re, this increase may not be monotonic. However, the variations in the damping rate are relatively small.

The same procedure can be applied for an elastic plate covering the free surface by merely modifying the representation of $\eta$ to take care of the additional boundary condition that has to be applied on the plate boundary.

Acknowledgements I thank the four anonymous referees for their valuable comments and criticism; these have led to a much improved paper. The work is funded by the Aeronautical Research \& Development Board, India under project number 1031452.

\section{Appendix}

$$
\begin{aligned}
& \beta_{n l}^{1,2}=\beta_{n l}^{4,5}=\int_{0}^{1} r p_{n}^{1,2}(r) J_{m}\left(\delta_{l} r\right) \mathrm{d} r, \quad \beta_{n l}^{3}=-\tanh \frac{\lambda_{n} h}{2} \int_{0}^{1} r p_{n}^{3}(r) J_{m}\left(\delta_{l} r\right) \mathrm{d} r, \\
& \xi_{n l}^{1,2}=\xi_{n l}^{4,5}=\int_{0}^{1} r q_{n}^{1,2}(r) J_{m}\left(\delta_{l} r\right) \mathrm{d} r, \quad \xi_{n l}^{3}=-\tanh \frac{\mu_{n} h}{2} \int_{0}^{1} r q_{n}^{3}(r) J_{m}\left(\delta_{l} r\right) \mathrm{d} r,
\end{aligned}
$$




$$
\begin{aligned}
& \chi_{n l}^{1,2}=\chi_{n l}^{4,5}=\int_{0}^{1} r s_{n}^{1,2}(r) J_{m}\left(\delta_{l} r\right) \mathrm{d} r, \quad \chi_{n l}^{3}=-\tanh \frac{\nu_{n} h}{2} \int_{0}^{1} r s_{n}^{3}(r) J_{m}\left(\delta_{l} r\right) \mathrm{d} r, \\
& \gamma_{n l}^{1,2}=-\beta_{n l}^{1,2}, \quad \rho_{n l}^{1,2}=-\xi_{n l}^{1,2}, \quad \psi_{n l}^{1,2}=-\chi_{n l}^{1,2}, \quad \gamma_{n l}^{4,5}=\beta_{n l}^{1,2}, \quad \rho_{n l}^{4,5}=\xi_{n l}^{1,2}, \quad \psi_{n l}^{4,5}=\chi_{n l}^{1,2}, \\
& \gamma_{n l}^{3}=-\left(\operatorname{coth}^{2} \lambda_{n} h / 2\right) \beta_{n l}^{3}, \quad \rho_{n l}^{3}=-\left(\operatorname{coth}^{2} \mu_{n} h / 2\right) \xi_{n l}^{3} \quad \psi_{n l}^{3}=-\left(\operatorname{coth}^{2} v_{n} h / 2\right) \chi_{n l}^{3}, \\
& \beta_{n l}^{6}=-\beta_{n l}^{3}, \quad \xi_{n l}^{6}=-\xi_{n l}^{3}, \quad \chi_{n l}^{6}=-\chi_{n l}^{3}, \quad \gamma_{n l}^{6}=-\gamma_{n l}^{3}, \quad \rho_{n l}^{6}=-\rho_{n l}^{3}, \quad \psi_{n l}^{6}=-\psi_{n l}^{3}, \\
& \theta_{n l}^{6}=\int_{0}^{1} r J_{m}\left(\delta_{n} r\right) J_{m}\left(\delta_{l} r\right) \mathrm{d} r, \quad \theta_{n l}^{7}=\theta_{n l}^{6}, \quad \theta_{n l}^{8}=\left(1+\tau \delta_{n}^{2}\right) \theta_{n l}^{6}, \\
& \beta_{n l}^{7}=\int_{0}^{1} r J_{m}\left(\lambda_{n} r\right) J_{m}\left(\delta_{l} r\right) \mathrm{d} r, \quad \xi_{n l}^{7}=\int_{0}^{1} r J_{m}\left(\mu_{n} r\right) J_{m}\left(\delta_{l} r\right) \mathrm{d} r, \quad \chi_{n l}^{7}=\int_{0}^{1} r J_{m}\left(v_{n} r\right) J_{m}\left(\delta_{l} r\right) \mathrm{d} r, \\
& \beta_{n l}^{8}=-\frac{2 \lambda_{n}}{\operatorname{Re}} \operatorname{coth} \frac{\lambda_{n} h}{2} \beta_{n l}^{3}, \quad \xi_{n l}^{8}=-\frac{2 \mu_{n}}{\operatorname{Re}} \operatorname{coth} \frac{\mu_{n} h}{2} \xi_{n l}^{3}, \quad \chi_{n l}^{8}=-\frac{2 v_{n}}{\operatorname{Re}} \operatorname{coth} \frac{\nu_{n} h}{2} \chi_{n l}^{3}, \\
& \gamma_{n l}^{7}=\beta_{n l}^{7}, \quad \rho_{n l}^{7}=\xi_{n l}^{7}, \quad \psi_{n l}^{7}=\chi_{n l}^{7}, \quad \gamma_{n l}^{8}=\beta_{n l}^{8}, \quad \rho_{n l}^{8}=\xi_{n l}^{8}, \quad \psi_{n l}^{8}=\chi_{n l}^{8} .
\end{aligned}
$$

\section{References}

1. Bauer HF, Chiba M (2007) Viscous oscillations in a circular cylindrical tank with elastic surface cover. J Sound Vib 304:1-17

2. Abramson HN (ed) (1966) The dynamic behaviour of liquid in moving containers. NASA SP 106

3. Bauer HF, Chiba M (2000) Hydroelastic viscous oscillations in a circular cylindrical container with an elastic cover. J Fluids Struct 14:917-936

4. Bauer HF, Chiba M (2005) Axisymmetric oscillation of a viscous liquid covered by an elastic structure. J Sound Vib 281:835-847

5. Kidambi R (2006) Oscillatory eddy structure in a cylindrical container. Fluid Dyn Res 38:274-294

6. Kidambi R (2009) Meniscus effects on the frequency and damping of capillary - gravity waves in a brimful circular cylinder. Wave Motion 46:144-154

7. Shankar PN (2007) Slow viscous flows : qualitative features and quantitative analysis using complex eigenfunction expansions. Imperial College Press, London

8. Howell DR, Buhrow B, Heath T, McKenna C, Hwang W, Schatz MF (2000) Measurements of surface-wave damping in a container. Phys Fluids 12(2):322-326

9. Martel C, Nicolás JA, Vega JM (1998) Surface-wave damping in a brimful circular cylinder. J Fluid Mech 360:213-228

10. Morse PM, Feshbach H (1953) Methods of theoretical physics, part 2. McGraw-Hill, New York

11. Shankar PN (2007) Frequencies of gravity - capillary waves on highly curved interfaces with edge constraints. Fluid Dyn Res 39:457-474

12. Nicolás JA (2002) The viscous damping of capillary-gravity waves in a brimful circular cylinder. Phys Fluids 14:1910-1919

13. Kidambi R (2007) Oscillations of a viscous free surface with pinned contact line. Fluid Dyn Res 39:121-138

14. Lamb H (1932) Hydrodynamics, 6th edn. Cambridge University Press, Cambridge

15. Bauer HF (1995) Coupled frequencies of a liquid in a circular cylindrical container with elastic liquid surface cover. J Sound Vib 180(5):689-704 